\title{
The integral equations of Compton Scatter Tomography
}

\author{
M. K. Nguyen \\ Equipes Traitement de l'Information et Systèmes (ETIS) \\ UMR CNRS 8051/ ENSEA / University of Cergy-Pontoise \\ F-95014 Cergy-Pontoise, France \\ mai.nguyen-verger@u-cergy.fr
}

\author{
T. T. Truong \\ Laboratoire de Physique Théorique et Modélisation (LPTM) \\ UMR CNRS 8089 / University of Cergy-Pontoise \\ F-95302 Cergy-Pontoise \\ truong@u-cergy.fr
}

\begin{abstract}
Two new Compton Scatter Tomography modalities, which are aimed at imaging hidden structures in bulk matter for industrial non-destructive control (or testing) and for medical diagnostics are shown to be based on the solutions of a special class of Chebyshev integral transforms. Besides their remarkable analytic properties, they can be inverted by existing methods which lend themselves nicely to numerical treatment and provide convergent, stable and fast computation algorithms. The existence of explicit inversion formulas implies that viable new imaging techniques can be developed, which may take over the current ones in a near future.
\end{abstract}

Keywords-Integral transforms; Radon transforms; Compton scatter imaging; Non-destructive testing; Medical imaging.

\section{Introduction}

Integral equations arise naturally in a wide range of fields as the proper way to represent a relevant phenomena to be exploited for applications. Examples are numerous in wave propagation, transport theory, optics, electromagnetism, acoustics, etc. Quite often, the conversion of differential processes into integral processes turns out to be beneficial in the sense that a solution can be worked out by integral operator techniques. This is in particular the case of aerodynamics, in which, for the first time an integral equation of the first kind appears with a Chebyshev integral kernel. Ta $\mathrm{Li}$ [1], who discovered this class of integral equations, has also realized that the equation he had discovered belongs to a much larger family with a hypergeometric function kernel, including numerous special cases involving Legendre, Jacobi, Gegenbauer polynomial kernels. What should be pointed out is the fact that their inversion, essential for applications, relies on a very peculiar property of the Gauss hypergeometric function, which was most elegantly derived by $R$. Buschman in [2].

In this paper, we show how the process of measuring energy flux densities of scattered gamma rays by bulk matter leads to the reconstruction its structure and composition without having to dismember it or take it apart. The image is called tomographic when such operation is done in a two-dimensional slice, perpendicular to some main axis of the object. A three-dimensional image can be then obtained by juxtaposition of a large number of tomographic images. To this end, in the next section, we recall the basic principle of Compton Scatter Tomography and introduce two recently suggested operating modalities. Section 3 handles the resulting integral transforms by describing the derivation of their inversion. The paper ends with a short conclusion and some perspectives for applications.

\section{Compton Scatter Tomography}

One of the most efficient way of probing the inner structure of an objects the use of penetrating radiation (Xor gamma-rays). Originally one exploits the phenomena of radiation attenuation inside matter from emission to detection along a straight line path. This has given rise to the widely known X-ray scanner, which is nowadays commonly used in hospitals and in industrial protocols. However as radiation propagates inside matter, scattering with distributed electric charges - or Compton effect - is the main cause for its attenuation. So a smart idea would be to collect information carried by scattered radiation to try to image the internal structure of matter. Of course there are many ways to put this idea to work. One of them is called Compton Scatter Tomography (or CST).

In CST the object is illuminated by a monochromatic source of penetrating radiation $\mathbf{S}$, placed at some spatial position. An energy sensitive detector $\mathbf{D}$ will measure the radiation flux density for a given scattered energy at all accessible sites outside the object. If the totality of such measurements allows to reconstruct the electric charge density inside the object, a corresponding image of this object is therefore obtained. This is the principle of CST, when this is done in a plane containing the line SD. As the object electric charge density is a smooth real-valued function $f(x, y)$ with compact support in $\mathrm{R}^{2}$, the set of measurements is required to have two independent variables. One natural variable is the radiation scattered energy, which is directly related to the scattering angle $\omega$ by the wellknown Compton relation. So when D registers scattered 
radiation at fixed energy and position in space, this means that it collects the scattered radiation flux density from all the scattering sites lying on a circular arc subtending an angle $(\pi-\omega)$, provided that selected collimators are set up so as to force the radiation rays to be inside a fixed plane. Hence the measurement at $\mathbf{D}$ is essentially an integral of $f(x, y)$ along this circular arc starting from $\mathbf{S}$ and ending at $\mathbf{D}$. The second natural variable to be taken is simply the angular position of SD with respect to a fixed reference direction. It may be labeled by an angle $\varphi$, i.e. The data registered at $\mathbf{D}$ is thus a function $g(\omega, \varphi)$. Omitting all unnecessary quantities, the mathematical problem at hand may be formulated as

$$
g(\omega, \varphi)=\int d x d y \delta(C) f(x, y)
$$

So for all allowable values of $(\omega, \varphi)$, how can one reconstruct the electric charge density $f(x, y)$ of an object from the data $g(\omega, \varphi)$ ? This is precisely the problem solved by J. Radon in 1917 for straight lines in a plane [3]. But here the same problem is posed for circular arcs $C$ going from $\mathbf{S}$ to $\mathbf{D}$. (1) has the structure of an integral equation of the first kind with a delta-function kernel $\delta(C)$ concentrated on $C$. Such a problem is usually ill-posed and occurs recurrently in imaging theory.

Let $\Gamma_{\mathrm{p}}$ be a circle of radius $p$, centered at the coordinate system origin $\mathrm{O}$. We suggest two ways of varying the relative positions of $\mathbf{S}$ and of $\mathbf{D}$ on $\Gamma_{\mathrm{p}}$, such that the inversion problem of this type of Radon transform can be solved exactly.

In modality 1 , the circular $\operatorname{arcs} C_{I}$ are inside $\Gamma_{\mathrm{p}}$, and the segment SD is a rotating diameter of $\Gamma_{\mathrm{p}}$ [4]. Thus the radiation source and the detector move rigidly around the apparatus center $\mathbf{O}$. This imposes some restrictions on the positioning of the object inside the scanning zone, unless the value of the parameter $p$ is changed.

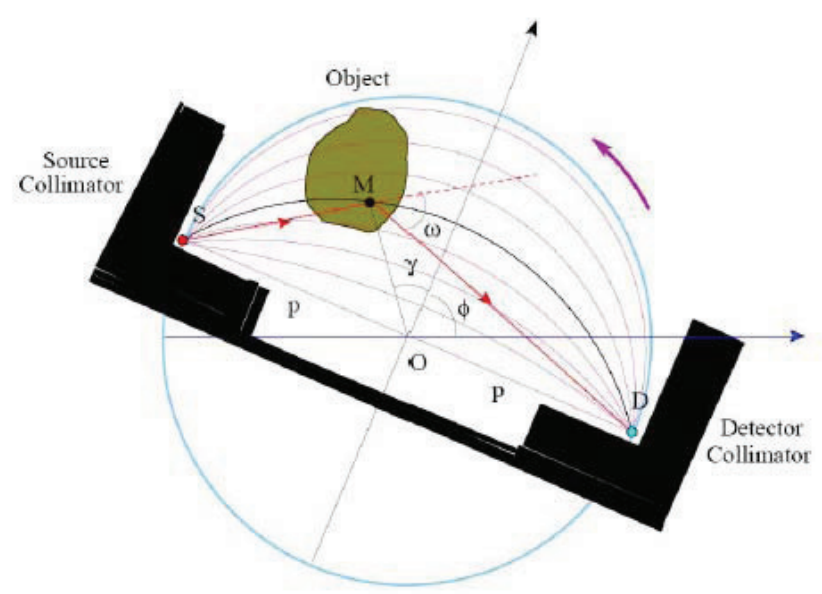

Fig.1 CST modality 1.
In modality 2 , the circular $\operatorname{arcs} C_{2}$ are also inside $\Gamma_{\mathrm{p}}$, and they intersect $\Gamma_{\mathrm{p}}$ at right angles [5]. The source $\mathbf{S}$ and the detector $\mathbf{D}$ are no longer at a constant distance from each other. They are separated on $\Gamma_{\mathrm{p}}$ by an angle $2 \gamma_{0}$ which is linked to the scattering angle $\omega$ by $\omega=\left(\gamma_{0}+\pi / 2\right)$. This gives a larger flexibility for positioning objects inside the scanning zone as compared to the previous modality.

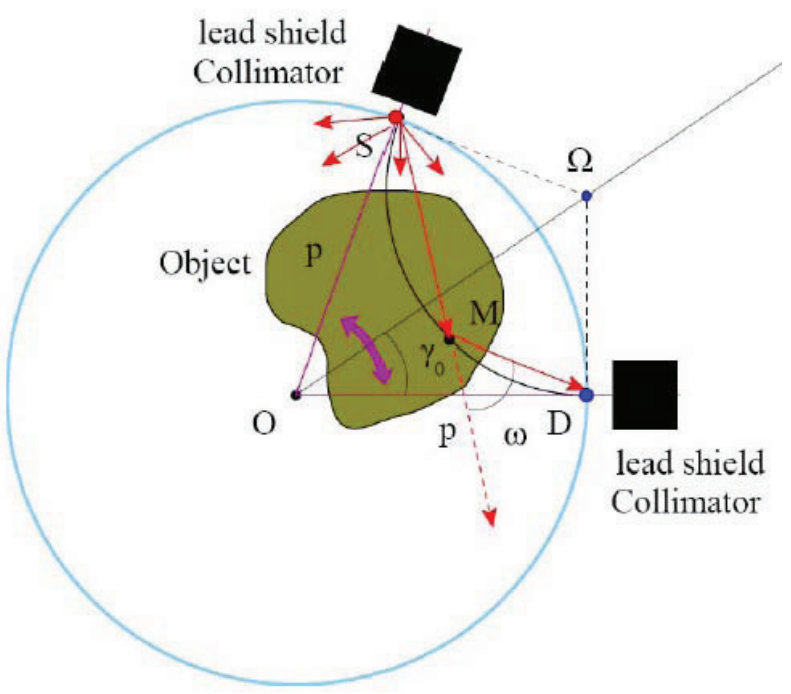

Fig. 2 CST modality 2

\section{Chebyshev Integral Transforms}

Since there is no preferred physical orientation in the plane, it is advantageous to work in polar coordinates and decompose the unknown function and its transform into angular Fourier series

$$
f(r, \theta)=\sum_{l=-\infty}^{\infty} f_{l}(r) e^{i l \theta} \text {, and } g(\omega, \varphi)=\sum_{l=-\infty}^{\infty} g_{l}(\omega) e^{i l \phi} .
$$

Then, for both modalities, (1) reduces to an ordinary integral equation of the first kind with a Chebyshev kernel for the Fourier components. Let $T_{l}(\mathrm{x})$ be the Chebyshev polynomial of the first kind or order $l$. Respectively we have

$$
\frac{\tau g_{l}^{-}(\tau)}{2 \sqrt{1+\tau^{2}}}=\int_{p\left(\sqrt{1+\tau^{2}}-\tau\right)}^{p} \frac{d r}{\sqrt{1-\frac{1}{4 \tau^{2}}\left(\frac{p}{r}-\frac{r}{p}\right)^{2}}} T_{l}\left(\frac{1}{2 \tau}\left(\frac{p}{r}-\frac{r}{p}\right)\right) f_{l}(r)
$$

for modality 1 , with $\tau=\cot \omega$, and

$$
\frac{\tau g_{l}^{+}(\tau)}{-2 \sqrt{\tau^{2}-1}}=\int_{p\left(\tau-\sqrt{\left.\tau^{2}-1\right)}\right.}^{p} \frac{d r}{\sqrt{1-\left(\frac{1}{2 \tau}\left(\frac{p}{r}+\frac{r}{p}\right)\right)^{2}}} T_{l}\left(\frac{1}{2 \tau}\left(\frac{p}{r}+\frac{r}{p}\right)\right) f_{l}(r)
$$


(4)

for modality 2 , with $\tau=\operatorname{cosec} \omega$.

To get their inverses we bring these equations to the form of the Chebyshev transform of A. M. Cormack [6] through the following change of variables

$$
q_{-}=\frac{1}{2}\left(\frac{p}{r}-\frac{r}{p}\right) \text {, and } q_{+}=\frac{1}{2}\left(\frac{p}{r}+\frac{r}{p}\right)
$$

(5)

and functions

$G_{l}^{-}(\tau)=\frac{\tau g_{l}^{-}(\tau)}{\sqrt{\tau^{2}+1}}$ and $F_{l}^{-}\left(q_{-}\right)=-\frac{p\left(\sqrt{q_{-}^{2}+1}-q_{-}\right) f_{l}\left(p\left(\sqrt{q_{-}^{2}+1}-q_{-}\right) \text {where } U_{l-l}(x) \text { is the Chebyshev polynomial of the second }\right.}{\sqrt{q_{-}^{2}+1}} \quad \begin{aligned} & \text { Using the Fourier series }\end{aligned}$

for modality 1 ,

$$
F^{ \pm}\left(q_{ \pm}, \theta\right)=\sum_{l \in \mathbb{Z}} F_{l}^{ \pm}\left(q_{ \pm}\right) e^{i l \theta}, \text { and } G^{ \pm}(\tau, \varphi)=\sum_{l \in \mathbb{Z}} G_{l}^{ \pm}(\tau) e^{i l \varphi \varphi}
$$

$G_{l}^{+}(\tau)=\frac{\tau g_{l}^{+}(\tau)}{\sqrt{\tau^{2}-1}}$ and $F_{l}^{+}\left(q_{+}\right)=\frac{p\left(\sqrt{q_{+}^{2}-1}+q_{+}\right) f_{l}\left(p\left(\sqrt{q_{+}^{2}-1}\right)+q_{+}(12)\right.}{\sqrt{q_{+}^{2}-1}}$ we reco

for modality 2 .

Then $(3,4)$ are rewritten as

$$
G_{l}^{ \pm}(\tau)=2 \int_{\sigma}^{\tau} d q_{ \pm} \frac{T_{l}\left(\frac{q_{ \pm}}{\tau}\right)}{\sqrt{1-\left(\frac{q_{ \pm}}{\tau}\right)^{2}}} F_{l}^{ \pm}\left(q_{ \pm}\right)
$$

which has the form of the Chebyshev transform in [6]. Here $\sigma=0,1$ for modality 1,2 .Thanks to the key formula $[2,6]$

$$
\int_{t}^{s} d q \frac{\cosh \left(l \cosh ^{-1}\left(\frac{q}{t}\right)\right)}{q \sqrt{\left(\frac{q}{t}\right)^{2}-1}} \frac{\cos l\left(\cos ^{-1}\left(\frac{q}{s}\right)\right)}{\sqrt{1-\left(\frac{q}{s}\right)^{2}}}=\frac{\pi}{2}
$$

the inversion of (8) can be achieved by a clever application of (9). The resulting explicit inversion formula reads

$$
F_{l}^{ \pm}\left(q_{ \pm}\right)=\frac{1}{\pi} \frac{d}{d q_{ \pm}} \int_{\sigma}^{q_{ \pm}} d \tau \frac{\cosh \left(l \cosh ^{-1}\left(\frac{q_{ \pm}}{\tau}\right)\right)}{\tau \sqrt{\left(\frac{q_{ \pm}}{\tau}\right)^{2}-1}} G_{l}^{ \pm}(\tau)
$$

However (10), as such is still improper for setting up a computational algorithm. It needs to be regularized as indicated in [7]. This is so because the transforms $G_{l}(\tau)$ have to verify the co-called consistency conditions, which consist Then the proper form of (10) is made up of two parts

$$
\begin{gathered}
F_{l}^{ \pm}\left(q_{ \pm}\right)=\frac{1}{\pi q_{ \pm}} \\
{\left[\int_{\sigma}^{q_{ \pm}} d \tau G_{l}^{\prime \pm}(\tau) \frac{\left(\frac{q_{ \pm}}{\tau}-\sqrt{\left(\frac{q_{ \pm}}{\tau}\right)^{2}-1}\right)^{l}}{\sqrt{\left(\frac{q_{ \pm}}{\tau}\right)^{2}-1}}-\int_{q_{ \pm}}^{\infty} d \tau G_{l}^{\prime \pm}(\tau) U_{l-1}\left(\frac{q_{ \pm}}{\tau}\right)\right]}
\end{gathered}
$$
of the vanishing of a finite number of moments of the data. we reconstruct the functions

$$
\begin{gathered}
F^{ \pm}\left(\frac{1}{2}\left(\frac{p}{r} \pm \frac{r}{p}\right), \theta\right)=( \pm) \frac{2 r}{\left(\frac{p}{r} \mp \frac{r}{p}\right)} f(r, \theta) \\
G^{ \pm}(\tau, \varphi)=\frac{\tau}{\sqrt{\tau^{2} \mp 1}} g^{ \pm}(\tau, \varphi)
\end{gathered}
$$

Then (11) may be used to derive a close form for the inversion formula first for the intermediate functions given in (12)

$$
F^{ \pm}\left(q_{ \pm}, \theta\right)=\frac{1}{2 \pi^{2} q_{ \pm}} \int_{\mathbb{S} 1} d \varphi \int_{\sigma}^{\infty} d \tau \frac{1}{\left(\frac{q_{ \pm}}{\tau}\right)-\cos (\theta-\varphi)} \frac{\partial}{\partial \tau} G^{ \pm}(\tau, \varphi)
$$

A final form of the inversion formula can be given in terms of the original functions $(f(r, \theta)$, the object electric charge density and $g(\tau, \varphi)$ the measured radiation flux density on the detector) by "extracting" $f(r, \theta)$ and $g(\tau, \varphi)$ from (13)

$$
\begin{gathered}
f(r, \theta)=( \pm) \frac{1}{2 \pi^{2} r} \frac{\left(\frac{p}{r} \mp \frac{r}{p}\right)}{\left(\frac{p}{r} \pm \frac{r}{p}\right)} \\
\int_{\mathbb{S} 1} d \varphi \int_{\sigma}^{\infty} d \tau \frac{1}{\frac{1}{2 \tau}\left(\frac{p}{r} \pm \frac{r}{p}\right)-\cos (\theta-\varphi)}\left(\frac{\partial}{\partial \tau} \frac{\tau g^{ \pm}(\tau, \varphi}{\sqrt{\tau^{2} \mp 1}}\right)
\end{gathered}
$$


(15)

In (15) the $\tau$-integral should be understood as a Cauchy principal value. The structure of this formula is analogous to that of the classical Radon transform. Yet it belongs to two types of Radon transforms on finite circular arcs which have appeared for the first time in the literature of integral geometry in the sense of I. M. Gelfand. This is in contrast to other types of Radon transforms which are defined on closed (or unbounded open) curves in the plane.

\section{Remark:}

In practice a computing algorithm can be set up from (11) following the approach of Chapman and Carey [9]. It consists in cutting the $\tau$-integration range into a finite number of integrals and then making the proper change of variables so that each term is represented by an exactly calculated indefinite integral. The derivative of $G_{l}(\tau)$ is simply calculated by linear interpolation. Of course the discretization steps must be optimized to save computing time. This way of reconstructing the unknown function turns out to be efficient, consistent with the data and give satisfactory results [10].

\section{Conclusion and Perspectives}

The idea of CST goes back to the early 50's. However the proposed scanning processes operate either point by point or line by line. Much later, to improve sensitivity, wide angle radiation collimators have been introduced. This has led naturally to the concept of integral measurements along arcs of circles. But no true inversion method was then at hand.

In the mid 90's, a first true CST modality was introduced by S. J. Norton [8]. It has a fixed source of radiation and a moving detector running on a straight line containing the source site. Such a CST scanner is, in particular appropriate for large objects which can only be studied from one side (such as a wall or a long metal beam). Here one may also formulate the problem in terms of circular harmonic components of the unknown electron density and end up with an integral equation with a Chebyshev kernel as shown independently by A. M. Cormack in [6]. The inversion procedure is thus simpler because one actually ends up with (14), in which $q_{+}$or $q_{\text {- }}$ is replaced by $q$.

Consequently the two CST scanning modalities proposed here appear to be complementary to Norton's CST modality. All three use Compton scattered radiation as imaging agent to probe the hidden parts of objects of interest in non-destructive control or in medical diagnostics. All three are based on the inversion of a Chebyshev integral transform with different degrees of complexity but share many common features which usher them into a near future as tomorrow imaging scanners for medical as well as industrial applications.

\section{REFERENCES}

[1] Ta. Li, "A new class of integral transforms," Proc. Amer. Math. Soc., vol. 11, pp. 290-298, 1960.

[2] R.G. Buschman, "Integrals of hypergeometric functions," Math. Zeitschr. vol 89, pp. 74-76, 1965.

[3] J. Radon, "Über die Bestimmung von Funktionnen durch ihre Integralwerte längst gewisser Mannigfaltigkeiten," Ber.Verh.Sachs.Akad.Wiss. Leipzig-Math.-Natur.K1. vol. 69, pp. 262-277, 1917.

[4] M. K. Nguyen and T. T. Truong, "Inversion of a new circular arc Radon transform for Compton scattering tomography," Inverse Problems vol. 26, pp. 065005, 2010.

[5] T. T. Truong and M. K. Nguyen, "Radon transforms on generalized Cormack's curves and a new Compton scatter tomography modality," Inverse Problems, vol. 27, pp. 125001, 2011.

[6] A. M. Cormack, "The Radon transform on a family of curves in the plane ," Proc. Amer. Math. Soc., vol. 83, pp. 325-330, October 1981.

[7] A. M. Cormack, "Radon's problem - Old and new", SIAM Proceedings, vol. 14, pp. 33-39, 1984.

[8] S. J; Norton, "Compton scattering tomography," J. Appl. Phys., vol. 70, pp. 2007-2015, 1994.

[9] C. H. Chapman and P. W. Carey, "The circular harmonic Radon transform," Inverse Problems, vol. 2, pp. 23-79, 1986.

[10] M. K. Nguyen, C. Faye, G. Rigaud and T. T. Truong, "A novel technological imaging process using ionizing radiation properties,"

Proceedings of the 9th IEEE - RVIF International Conference on Computing and Communications Technologies (RIVF 12), Ho-chi-Minh City, Vietnam, February 2012. 